\title{
Direct Constraints on the Impact of TP-AGB Stars on the SED of Galaxies from Near-Infrared Spectroscopy
}

\author{
Stefano Zibetti ${ }^{1}$, Anna Gallazzi ${ }^{1}$, Stéphane Charlot ${ }^{2}$, Anna Pasquali ${ }^{3}$ \\ and Daniele Pierini $\dagger$ \\ ${ }^{1}$ Dark Cosmology Centre, Niels Bohr Institute, University of Copenhagen, \\ Juliane Maries Vej 30, DK-2100 Copenhagen, Denmark \\ email: zibetti, gallazzi @dark-cosmology.dk \\ ${ }^{2}$ Institut d'Astrophysique de Paris, France \\ ${ }^{3}$ Astronomisches Rechen-Institut, Heidelberg, Germany
}

\begin{abstract}
We present new spectro-photometric NIR observations of 16 post-starburst galaxies especially designed to test for the presence of strong carbon features of thermally pulsing AGB (TP-AGB) stars, as predicted by recent models of stellar population synthesis. Selection based on clear spectroscopic optical features indicating the strong predominance of stellar populations with ages between 0.5 and $1.5 \mathrm{Gyr}$ and redshift around 0.2 allows us to probe the spectral region that is most affected by the carbon features of TP-AGB stars (unaccessible from the ground for $z \sim 0$ galaxies) in the evolutionary phase when their impact on the IR luminosity is maximum. Nevertheless, none of the observed galaxies display such features. Moreover the NIR fluxes relative to optical are consistent with those predicted by the original Bruzual \& Charlot (2003) models, where the impact of TP-AGB stars is much lower than has been recently advocated.
\end{abstract}

Keywords. galaxies: general - galaxies: galaxies: stellar content galaxies: fundamental parameters - infrared: galaxies, stars - stars: AGB and post-AGB.

\section{TP-AGB Stars: the big unknown}

The modeling and interpretation of the spectral energy distribution (SED) of stellar populations via stellar population synthesis (SPS) is a fundamental tool to understand galaxy properties and their evolution. Yet we are far from a complete comprehension and a reliable modeling of some stellar evolutionary phases which strongly affect the energy output of stellar populations. Among them, the so-called thermally pulsing - asymptotic giant branch (TP-AGB for short) phase has been the focus of debate among modelists for several years (e.g. Maraston 2005; Bruzual 2007; Marigo et al. 2008). TP-AGB stars are believed to be major contributors to the NIR flux of stellar populations with age between 0.5 and 1.5 Gyr. However, quantitative predictions of the lifetimes, luminosities and emission continuum shapes and spectral features of these stars and of their influence on the integrated spectra of even "simple" stellar populations, are still very uncertain and strongly model-dependent. Different treatments of TP-AGB stars (i.e. prescriptions to model their lifetimes and luminosities, different stellar spectral libraries) in popular SPS codes can yield significant differences, in particular concerning $i$ ) the flux ratios in different NIR bands and in NIR vs optical bands and ii) the presence (or absence) of sharp spectral features due to molecules in the atmospheres of those stars. The age and spectral ranges affected by TP-AGB stars are of particular interest for two main applications. a)

$\dagger$ Visitor astronomer at the Max-Planck-Institut für extraterrestrische Physik, Garching 
At low redshift, the NIR is often used as tracer of stellar mass in galaxies, both unresolved (e.g. Bell et al. 2003) and resolved ones (e.g. Zibetti, Charlot \& Rix, 2009), because of the (supposed) lower sensitivity to the young stellar components that often dominate the visible part of the spectrum and because of lower dust extinction. However, different treatments of TP-AGB stars in SPS models result in systematic differences in global stellar mass estimates of several tens of percent and in substantially different estimates of the stellar mass density contrast of structures like spiral arms within galaxies (see Zibetti, Charlot \& Rix, 2009). b) At $z \gtrsim 2.5$, the bulk of stellar populations has mean ages in the range 0.5-1.5 Gyr that is (allegedly) mostly affected by TP-AGB stars. As demonstrated by, e.g., Maraston et al. (2006) and Kannappan \& Gawiser (2007), uncertainties on TP-AGB models severely affect stellar mass and age determinations for galaxies at those redshifts (see also Conroy, Gunn \& White, 2009).

\section{Testing SPS model predictions: NIR spectrophotometric observations of post-starburst galaxies at $z \sim 0.2$}

We directly test the impact of TP-AGB stars on the integrated SED of galaxies by obtaining spectra in $\mathrm{H}$ and $\mathrm{K}$ bands, which we combine with available optical spectroscopy and photometry. Such an SED can be confronted with SPS predictions and used to constrain model templates for galaxies at low and high redshifts. In order to maximize the impact of TP-AGB stars, we target "post-starburst" galaxies, whose star formation an intense burst 0.5-1.5 Gyr back in the past, followed by negligible activity. They can be relatively easily selected from the large SDSS spectral database by requiring no emission in $\mathrm{H} \alpha$ or in [OII] and equivalent width of $\mathrm{H} \delta>5 \AA$ in absorption (following Goto 2005). In addition we require light-weighted mean stellar age between 0.5 and 1.5 Gyr as determined from optical spectral indexes (little sensitive to TP-AGB modeling) according to the bayesian method of Gallazzi et al. (2005). As the most prominent NIR features of TP-AGB stars are located at 1.41 and $1.77 \mu \mathrm{m}$ and, thus, fall in the atmospheric gaps between $\mathrm{J}$ and $\mathrm{H}$, and $\mathrm{H}$ and $\mathrm{K}$ bands for $z \sim 0$ galaxies, we selected galaxies at $z \sim 0.2$, so that those two features move to the middle of the $\mathrm{H}$ and $\mathrm{K}$ atmospheric windows, respectively. The resulting sample of 16 galaxies provides a good coverage of the age-metallicity domain that is most strongly affected by TP-AGB stars.

NIR spectra in $\mathrm{H}$ and $\mathrm{K}$ bands have been obtained with ISAAC at the ESO-VLT during P86 and assembled with the SDSS spectra to provide flux-calibrated SEDs ranging from $\sim 3000$ to $\sim 21000 \AA$ with a rest-frame resolution of $3 \AA$ in the optical and $40 \AA$ in the NIR and typical $\mathrm{S} / \mathrm{N}>30$ per resolution element (see examples in Fig. 11). Spectra have been rescaled to match the broad-band "total" fluxes derived from SDSS, UKIDSS and ISAAC imaging in matched apertures corresponding to the SDSS Petrosian aperture.

Where are the TP-AGB NIR spectral features? The H- and K-band spectra of all 16 galaxies in the sample appear smooth and featureless. In particular we do not detect either of the two sharp features at $1.41 \mu \mathrm{m}$ and $1.77 \mu \mathrm{m}\left(\mathrm{C}_{2}\right)$ rest-frame, in clear contrast with the Maraston (2005) models predicting extremely strong and abrupt flux drops of 10 to $30 \%$ at those wavelengths. Our observations are the first ones to prove this directly thanks to the NIR spectroscopy and the choice of a suitable redshift range. Nine examples out of our 16 observed galaxies are reported in Fig. 1 to demonstrate the lack of such NIR spectral features. Spanning the full range of metallicities over the age range where TP-AGB stars are expected to peak, there is no sign of sharp NIR spectral features.

"TP or not TP? that is the question" Over the last few years a general consensus has grown among SPS modelists about the need for a boosted NIR flux due to TP-AGB stars with respect to earlier generation models (e.g. BC03), although with strong differences 


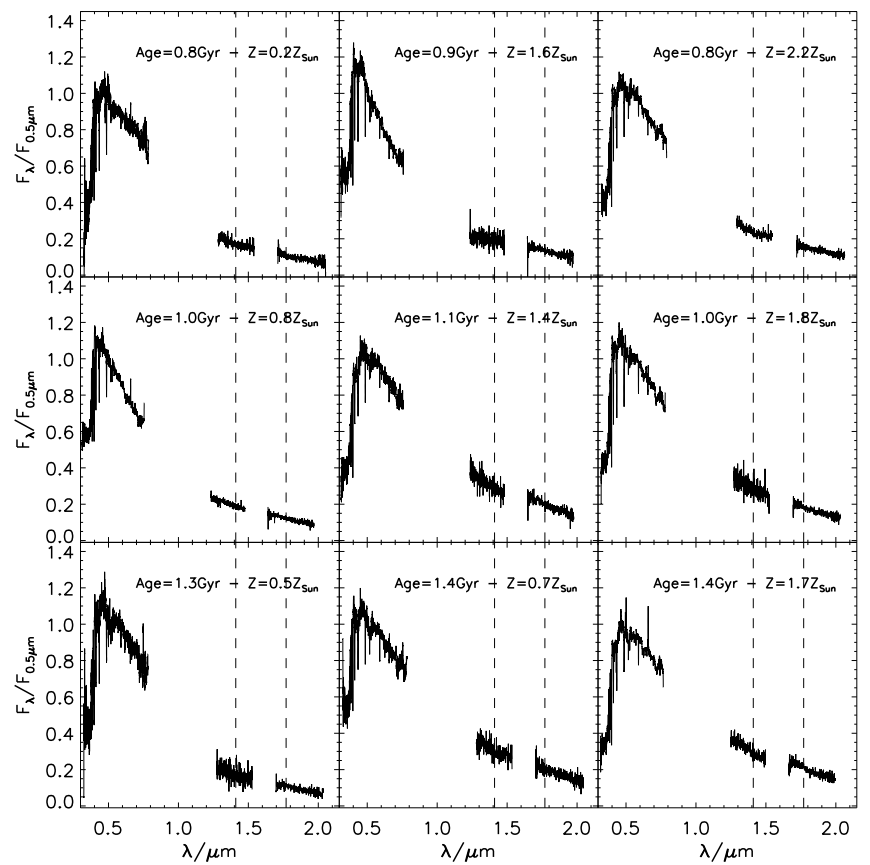

Figure 1. Nine examples of combined optical-NIR spectra (ISAAC for the NIR, SDSS for the optical) of the post-starburst galaxies in our sample. The three rows show galaxies of increasing mean light-weighted age, from $\sim 0.8$ Gyr (top), to $\sim 1$ Gyr (middle) to $\sim 1.4$ Gyr (bottom). In each row light-weighted mean metallicity increases from left to right, from sub-solar to super-solar values. The spectra in each panel are cross-calibrated based on SDSS, UKIDSS and ISAAC photometry normalized to the flux at $0.5 \mu \mathrm{m}$ (rest) and shown as a function of rest-frame wavelength. The vertical dashed lines mark 1.41 and $1.77 \mu \mathrm{m}$, where sharp spectral features are expected based on the Maraston (2005) models (cf. their Fig. 14, 15 and 18). However neither is observed. Each panel is labeled with the light-weighted stellar age and metallicity derived using the bayesian method of Gallazzi et al. (2005).

concerning its exact amount. Kriek et al. (2010) have recently challenged this, by showing that the composite (medium-band NIR) SED of 62 post-starburst galaxies at $0.7<$ $z<2.0$ is fully consistent with BC03 and inconsistent with Maraston (2005). Although very careful analysis is needed to compare our new observations with different models (in prep.), preliminary analysis shows that the majority of our spectra is extremely well reproduced by BC03 simple stellar populations (SSP) with no need for further tweaking/fitting or inclusion of dust. This suggests that predictions from early models might not require very substantial correction in terms of NIR output.

\section{References}

Bell, E.F., McIntosh, D.H., Katz N., Weinberg, M.D. 2003, ApJS, 149, 289

Bruzual, G. 2007, arXiv:astro-ph/0703052

Bruzual, G. \& Charlot, S. 2003, MNRAS, 344, 1000

Conroy, C., Gunn, J.E., White, M. 2009, ApJ, 699, 486

Gallazzi, A., et al. 2005, MNRAS, 362, 41

Goto, T. 2005, MNRAS, 357, 937

Kannappan, S.J. \& Gawiser, E. 2007, ApJ, 657, L5

Kriek, M., et al. 2010, ApJL, 722, 64

Maraston, C. 2005, MNRAS, 362, 799

Maraston, C. et al. 2006, ApJ,652, 85

Marigo, P., et al. 2008, A\& $\mathcal{E} A, 482,883$

Zibetti, S., Charlot, S., Rix, H.-W. 2009, MNRAS, 400, 1181 